\title{
Effective Diversity of OTFS Modulation
}

\author{
P. Raviteja, Yi Hong, Emanuele Viterbo, and Ezio Biglieri
}

\begin{abstract}
Orthogonal time frequency space (OTFS) modulation, which encodes information symbols in the delay-Doppler domain, offers a promising solution to the problem of high Doppler sensitivity of orthogonal frequency division multiplexing (OFDM) transmission. In this paper we study the diversity of OTFS assuming rectangular waveforms and a delay-Doppler channel with two paths. After introducing the concept of $e f$ fective diversity (ED), which we argue to be more significant than "standard" diversity in the case of a large number of transmitted symbols, we examine the conditions under which OTFS achieves full ED for QAM symbols. We validate our analytical results through numerical simulations, which show that OTFS practically achieves full ED with sufficiently large signal constellations.
\end{abstract}

Index Terms-Delay-Doppler channel, diversity, OTFS.

\section{INTRODUCTION}

A simple and effective way of evaluating symbol error rates in digital communications is based on the concept of pairwise error probabilities (PEPs) [1], whose sum over all transmitted signal pairs yields an upper bound to said rate. With Rayleigh fading channels, performance is often expressed using a single parameter, called diversity, representing the minimum slope of the PEP-vs.-SNR curves across all signal pairs. This turns out to be the slope of the actual symbol error probability for high SNR. Now, in the case of a large number of transmitted signals and intermediate SNR, diversity may not be a meaningful parameter, as a large majority of PEP curves may exhibit a slope much steeper than the minimum. In these conditions, we advocate the use of an effective diversity (ED), which accounts for the slope of the majority of PEP curves rather than the minimum one. In this paper, we focus on studying this parameter for orthogonal time frequency space (OTFS) modulation, which is a recently proposed waveform for high Doppler channels [2], [3]. Some other works on OTFS may be found in [4]-[14].

Recently, in [8], the authors analyzed the conventional diversity of OTFS for ideal bi-orthogonal waveforms and showed that it is one. However, this analysis does not reflect the PEP values at practical SNR's and also it is not valid for practical waveforms. In this paper, we study the performance of OTFS using ED with practical rectangular waveforms and operating over a delay-Doppler channel with two paths. ED is derived, and numerical simulations are used to validate our results.

Raviteja, Hong, and Viterbo are with ECSE Department, Monash University, Clayton, VIC 3800, Australia, Email: \{raviteja.patchava, yi.hong, emanuele.viterbo\}@monash.edu. Biglieri is with Universitat Pompeu Fabra, Barcelona, Email: ezio.biglieri@gmail.com.

The work of the first three authors was supported by the Australian Research Council through the Discovery Project under Grant DP160100528. The work of Ezio Biglieri was supported by the European Research Council under the H2020 Framework Programme/ERC grant agreement 694974.
Notations: Scalars, vectors, and matrices are denoted $a$, a, and $\mathbf{A}$, respectively. $\mathbf{A}^{\dagger}$ and $\mathbf{A}^{i}$ denote Hermitian trans-pose and $i^{t h}$ power of $\mathbf{A}$. We write $\mathbf{a}=\operatorname{vec}(\mathbf{A})$ for the column-wise vectorization of matrix $\mathbf{A}$. Symbols $\mathbf{a}(i)$ and $\mathbf{A}(i, j)$ denote the $i^{\text {th }}$ element of $\mathbf{a}$ and $(i, j)^{\text {th }}$ element of $\mathbf{A}$, respectively. Further, $\mathbf{A}=\operatorname{diag}\left[a_{0}, \cdots, a_{N-1}\right]$ denotes a diagonal matrix of size $N$ with $\left\{a_{0}, \cdots, a_{N-1}\right\}$ as its diagonal elements. $\mathbf{I}_{N}$ is the

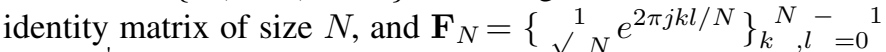
and $\mathbf{F}^{\dagger}{ }_{N}$ are the $N$-point DFT and the IDFT matrices, respectively. Symbol $\otimes$ denotes Kronecker product, and $\mathcal{C N}(\mathbf{0}$, $\left.N_{0} \mathbf{I}_{N}\right)$ an i.i.d. Gaussian random vector with mean $\mathbf{0}$ and covariance matrix $N_{0} \mathbf{I}_{N}$. Notation [ $\left.\cdot\right]_{N}$ denotes $\bmod -N$ operation, and $\operatorname{gcd}(a, b)$ the greatest common divisor of $a$ and $b$. Finally, $\mathbf{Z}$ and $\mathbf{Z}[j]$ denote the set of integers and the number field whose elements have the form $a+b j$, with $a$ and $b$ integers, respectively. Finally, $\mathbf{Q}\left(e^{-j 2 \pi}{ }_{N}{ }^{1}\right)$ denotes the cyclotomic field obtained by adjoining an $N$-th root of unity to the set of rational numbers.

\section{SYSTEM MODEL}

We consider OTFS modulation with one transmit and one receive antenna. Let $\mathbf{X} \in \mathbb{A}^{M \times N}$ denote the two-dimensional (QAM) information symbols in the delay-Doppler domain, where the QAM alphabet $\mathbb{A} \in\left\{a_{0}, \cdots, a_{Q-1}\right\}$. Note that $\mathbb{A} \subset$ $\mathbf{Z}[j]$. Assuming rectangular waveforms, the OTFS transmitted signal can be written as [5]

$$
\mathbf{s}=\operatorname{vec}\left(\mathbf{F}_{M}^{\dagger}\left(\mathbf{F}_{M} \mathbf{X} \mathbf{F}_{N}^{\dagger}\right)\right)=\left(\mathbf{F}_{N}^{\dagger} \otimes \mathbf{I}_{M}\right) \mathbf{x}
$$

where $\mathbf{x} \triangleq \operatorname{vec}(\mathbf{X}) \in \mathbb{A}^{M N}$. For efficient FFT implementation, we assume $M$ and $N$ are powers of 2 .

The received signal can be put in the form [5]

$$
\mathbf{r}=\mathbf{H s}+\mathbf{w},
$$

where, under the assumption that the channel admits a $P$-path sparse representation as described in [6], $\mathbf{H}$ is the $M N \times M N$ channel matrix

$$
\mathbf{H}=\sum_{i=1}^{P} h_{i} \boldsymbol{\Pi}^{l_{i}} \boldsymbol{\Delta}^{k_{i}},
$$

with $\Pi$ the permutation matrix (forward cyclic shift), $\Delta$ the $M N \times M N$ matrix $\boldsymbol{\Delta} \triangleq \operatorname{diag}\left[z^{0}, z^{1}, \cdots, z^{M N-1}\right], z \triangleq$ $e^{\frac{j 2 \pi}{M N}}$, and $\mathbf{w} \sim \mathcal{C N}\left(\mathbf{0}, N_{0} \mathbf{I}\right)$.

After OTFS processing, the received signal in delayDoppler domain becomes

$$
\begin{aligned}
\mathbf{y} & =\left(\mathbf{F}_{N} \otimes \mathbf{I}_{M}\right) \mathbf{H}\left(\mathbf{F}_{N}^{\dagger} \otimes \mathbf{I}_{M}\right) \mathbf{x}+\left(\mathbf{F}_{N} \otimes \mathbf{I}_{M}\right) \mathbf{w} \\
& =\mathbf{H}_{\text {eff }} \mathbf{x}+\widetilde{\mathbf{w}}
\end{aligned}
$$

where $\mathbf{H}_{\mathrm{eff}} \triangleq\left(\mathbf{F}_{N} \otimes \mathbf{I}_{M}\right) \mathbf{H}\left(\mathbf{F}_{N}^{\dagger} \otimes \mathbf{I}_{M}\right)$ is the effective channel matrix, and $\widetilde{\mathbf{w}}=\left(\mathbf{F}_{N} \otimes \mathbf{I}_{M}\right) \mathbf{w}$ the noise vector. 


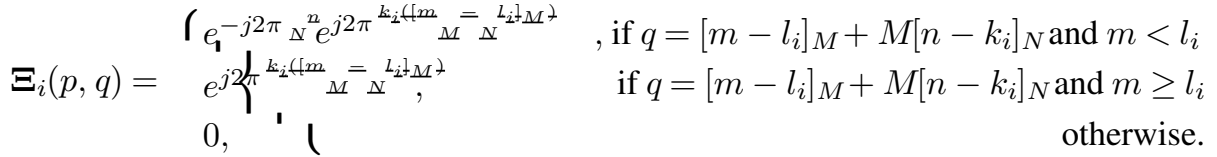

In order to study the diversity of OTFS, we use the following two observations from our earlier work [5], [6]. Observation 1. The received signal $y$ can be written as [6] $\mathbf{y}={ }_{i=1}^{P} \mathbf{k}_{i}\left(\mathbf{F}_{N} \otimes \mathbf{I}_{M}\right) \mathbf{\Pi}^{l_{i}} \boldsymbol{\Delta}^{k_{i}}\left(\mathbf{F}_{N}^{\dagger} \otimes \mathbf{I}_{M}\right) \mathbf{x}+\mathbf{w}^{\sim}=\boldsymbol{\Phi}(\mathbf{x}) \mathbf{h}+\mathbf{w}^{\sim}$ where $\mathbf{h}=\left[h_{1}, h_{2}, \cdots, h_{P}\right]$ is a $P \times 1$ vector of i.i.d. complex Gaussian random variables, and $\boldsymbol{\Phi}(\mathbf{x})$ is the $M N \times P$ concatenated matrix

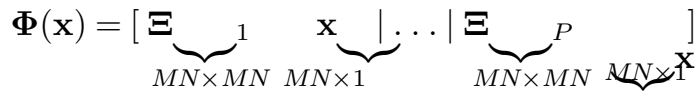

and $\boldsymbol{\Xi}_{i} \triangleq\left(\mathbf{F}_{N} \otimes \mathbf{I}_{M}\right) \boldsymbol{\Pi}^{l_{i}} \boldsymbol{\Delta}^{k_{i}}\left(\mathbf{F}_{N}^{\dagger} \otimes \mathbf{I}_{M}\right)$.

The conditional pairwise error probability for the above system can be written as

$$
\begin{aligned}
P\left(\mathbf{x} \rightarrow \mathbf{x}^{\wedge} \mid \mathbf{h}\right) & =\mathbb{P}\left(\left\|\quad \mathbf{y}-\mathbf{\Phi}\left(\mathbf{x}^{\wedge}\right) \mathbf{h}\right\|^{\mathbf{2}}<\|\mathbf{y}-\mathbf{\Phi}(\mathbf{x}) \mathbf{h}\|^{\mathbf{2}} \mid \mathbf{h}\right) \\
& =\mathcal{Q}\left(\begin{array}{c}
\|\left[\mathbf{\Phi}\left(\mathbf{x}^{\wedge}\right)=\right. \\
\sqrt{2 \Phi}(\mathbf{x})] \mathbf{h} \|
\end{array}\right)
\end{aligned}
$$

where $\mathcal{Q}$ is the Gaussian tail function.

Now, using the Chernoff upperbound, the pairwise error probability becomes

where

$$
P(\mathbf{x} \rightarrow \wedge \mathbf{x}) \leq \mathbb{E}_{\mathbf{h}} \exp \left(\begin{array}{c}
-\|\underline{\boldsymbol{\Phi}}(\boldsymbol{\delta}) \mathbf{h}\|^{2} \\
4 N_{0}
\end{array}\right)
$$

$$
\boldsymbol{\Phi}(\boldsymbol{\delta})=\left[\boldsymbol{\Xi}_{1} \boldsymbol{\delta}|\ldots| \boldsymbol{\Xi}_{P} \boldsymbol{\delta}\right]
$$

and $\boldsymbol{\delta}=\mathrm{x}^{\wedge}-\mathrm{x}$ is the codeword difference vector. Note that $\boldsymbol{\delta}$ $\subset \mathbf{Z}[j]^{M N \times 1}$.

Assuming $\mathbf{h} \sim \mathcal{C N}(\mathbf{0}, \mathbf{I})$ and $\boldsymbol{\Upsilon}(\boldsymbol{\delta})=\boldsymbol{\Phi}^{\dagger}(\boldsymbol{\delta}) \boldsymbol{\Phi}(\boldsymbol{\delta})$, the upper bound on the average bit error probability (BER) $P_{\mathrm{e}}$ can be written as [6, Eq. (23)]

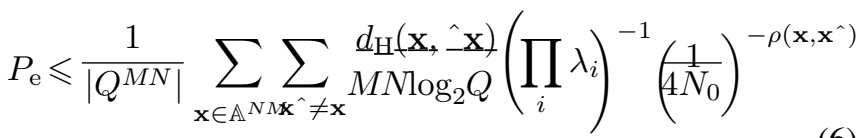

where, $M N \log _{2} Q$ denotes the total number of bits transmit-ted in one OTFS frame, $d_{\mathrm{H}}\left(\mathbf{x},{ }^{\wedge} \mathbf{x}\right)$ is the difference in number of information bits between $\mathbf{x}$ and $\mathbf{x}^{\wedge}, \lambda_{i}$ 's are the non-zero eigenvalues of $\mathbf{\Upsilon}(\boldsymbol{\delta})$, and $\rho\left(\mathbf{x}, \mathbf{x}^{\wedge}\right)$ is the number of nonzero eigenvalues of $\boldsymbol{\Upsilon}(\boldsymbol{\delta})$.

Finally, the "standard" definition of diversity of OTFS is

$$
\rho \triangleq \min _{\mathbf{x} \neq \mathbf{x}^{\wedge},\left(\mathbf{x}, \mathbf{x}^{\wedge}\right)} \rho\left(\mathbf{x}, \mathbf{x}^{\wedge}\right) \underset{\mathbf{x} \neq \mathbf{x}^{\wedge},\left(\mathbf{x}, \mathbf{x}^{\wedge}\right)}{\operatorname{rank}}(\mathbf{\Upsilon}(\boldsymbol{\delta}))
$$

Observation 2. In matrix $\boldsymbol{\Xi}_{i}$ as given in (8), where $0 \leq p \leq$ $M N-1$ and $0 \leq q \leq M N-1$ [5, Theorem 1], the values of $n$ and $m$ can be computed as $n=\left\lfloor{ }^{p}\right\rfloor$ and $m=p-n M$. Noth

that $\boldsymbol{\Xi}_{i} \boldsymbol{\delta}$ is equivalent to a circulant shift of $\boldsymbol{\delta}$ with some phase shifts. In next section, we derive the diversity order achieved by OTFS system for two channel paths, i.e., $P=2$.

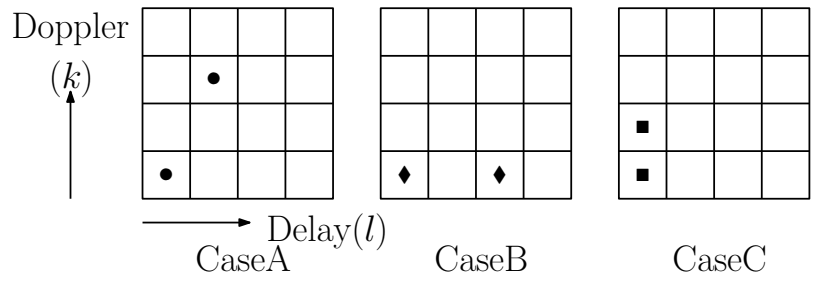

Fig. 1. Examples for the three cases in the diversity analysis for $M=N=4$

III. DIVERSITY ANALYSIS FOR $P=2$

Without loss of generality, we assume that the two channel paths are located at $\left(l_{1}, k_{1}\right)=(0,0)$ and at $\left(l_{2}, k_{2}\right)$. We divide the diversity analysis of OTFS into the following three cases: $A$. $l_{2} \neq 0$ and $k_{2} \neq 0, B . l_{2} \neq 0$ and $k_{2}=0, C . l_{2}=0$ and $k_{2} \neq 0$.

Fig. 1 describes the examples of the three cases for the OTFS system of $M=N=4$. Here, the dots represent the delay and Doppler positions of the two channel paths.

\section{A. $l_{2} \neq 0$ and $k_{2} \neq 0$}

Since there are no shifts across the delays or Dopplers due to the first path (i.e., $\boldsymbol{\Xi}_{1}=\mathbf{I}$ ), the first column of $\boldsymbol{\Phi}(\boldsymbol{\delta})$ in (5) can be written as

$\boldsymbol{\Phi}_{1}(\boldsymbol{\delta})=\boldsymbol{\delta}=\left[\delta_{0,0}, \delta_{1,0}, \cdots, \delta_{M-1,0}, \delta_{0,1}, \delta_{1,1}, \cdots, \delta_{M-1,1}\right.$,
$\left.\cdots, \delta_{0, N-1}, \delta_{1, N-1}, \cdots, \delta_{M-1, N-1}\right]^{T}(9)$

where $\delta_{m, n}$ denotes the $m+M n$ element of $\delta$. The second column of $\boldsymbol{\Phi}(\boldsymbol{\delta})$ is $\left(l_{2}, k_{2}\right)$ circulant shifted version of $\boldsymbol{\Phi}_{\mathbf{1}}(\boldsymbol{\delta})$ with some phase shifts as given in (8). Given that we exclude the case $\boldsymbol{\delta}=0\left(\mathbf{x} \neq \mathbf{x}^{\wedge}\right)$, we have at least one element $\delta_{m, n} \neq 0$ for some $m$ and $n$. Assume $m \geq l_{2}{ }^{1}$. Let us consider two rows in $\boldsymbol{\Phi}(\boldsymbol{\delta})$ with $\delta_{m, n}$ as one of the element:

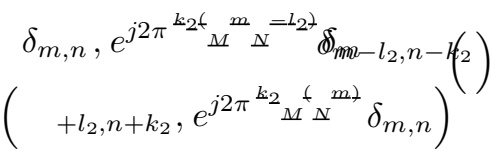

The second element in (10) can be made zero by multiplying $\delta_{m}$

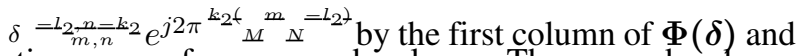
subtracting from second column. The second column entry in (11) becomes

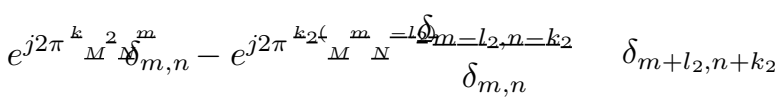

If we can show that the value in (12) is non-zero for all values of $\delta_{m, n} \in \mathbf{Z}[j]$, then we can say the two columns of $\boldsymbol{\Phi}(\boldsymbol{\delta})$ are independent for QAM modulation and we guarantee a diversity order 2 , which is the number $P$ of paths and hence the maximum achievable value.

\footnotetext{
${ }^{1}$ Note that for $m<l_{2}$, the expression in (12) will be similar except a phase shift of ${ }^{n} \bar{N}^{j} j \pi$ in the second term. Assume the value of (12) for $m<l_{2}$ is zero, i.e., $\delta_{m+l_{2}, n+k_{2}} \neq 0$. By repeating (12) for $\delta_{m+l_{2}, n+k_{2}}$, we can always get a condition $\delta_{m^{\prime}, n^{\prime}} \neq 0$, for which $m^{\prime}>l_{2}$.
} 
The value in (12) becomes zero if

$$
\delta_{m, n}^{2}=e^{-j 2 \pi \frac{k_{2} l_{2}}{M N}} \delta_{m-l_{2}, n-k_{2}} \delta_{m+l_{2}, n+k_{2}}
$$

If $l_{2} \neq 0$ and $k_{2} \neq 0$ then the value $e^{-j 2 \pi \frac{k_{2} l_{2}}{M N}}$ is irrational belonging to the cyclotomic field $\mathbf{Q}\left(e^{-j 2 \pi \frac{k_{2} l_{2}}{M N}}\right)$ of degree $\varphi\left(\min \left(M N, M N / k_{2} l_{2}\right)\right) \geq 2$ for any $k_{2}, l_{2}$ such that $k_{2} l_{2}<M N / 4$ and the above equality cannot be satisfied by $\delta_{n, m} \in \mathbf{Z}[j]$ (Here $\varphi(n)$ denotes the Euler totient function, counting the number of relatively primes to $n$, and the second argument of the min only applies for $k_{2} l_{2}$ dividing $M N$.)

Since the typical wireless channels are underspread, i.e., $l_{2} \ll M, k_{2} \ll N$, for $P=2$ paths at $(0,0)$ and $\left(l_{2}, k_{2}\right)$ with $l_{2} \neq 0$ and $k_{2} \neq 0$, we have diversity order 2 .

Example: For $M=N=4$ and $k_{2}=l_{2}=1$, the condition in (12) becomes

$$
\delta_{m, n}^{2}=e^{-j \pi \frac{1}{8}} \delta_{m-l_{2}, n-k_{2}} \delta_{m+l_{2}, n+k_{2}}
$$

Since (14) cannot be satisfied for $\delta_{n, m} \in \mathbf{Z}[j]$, we have diversity order of 2. $\square$ Special case: For $M=N=2$ and $k_{2}=$ $l_{2}=1$, the conditions in (10) and (11) becomes

$$
\delta_{, m^{m}=-j \delta^{2}}^{2} \quad\left[m-l_{2}\right]_{M},\left[n-k_{2}\right]_{N} \text { (or) } \delta_{,}^{2}, m=j \delta^{2} \quad\left[m-l_{2}\right]_{M},\left[n-k_{2}\right]_{N}
$$

However, for $\delta_{n, m} \in \mathbf{Z}[j]$, neither one of the above conditions can be satisfied, which yields full diversity.

\section{B. $l_{2} \neq 0$ and $k_{2}=0$}

Let us rewrite the $\boldsymbol{\Phi}(\boldsymbol{\delta})$ in (7) as follows:

$$
\boldsymbol{\Phi}(\boldsymbol{\delta})=\left[\begin{array}{ll}
\Xi_{1} & \Xi_{2}
\end{array}\right]\left[\begin{array}{ll}
\boldsymbol{\delta} & 0 \\
0 & \delta
\end{array}\right]
$$

Now, from (15), $\Upsilon(\boldsymbol{\delta})$ can be written in the form

$$
\Upsilon(\delta)=\Phi^{\dagger}(\delta) \Phi(\delta)=\left[\begin{array}{cc}
\delta^{\dagger} \delta & \delta^{\dagger} \Lambda \delta \\
\delta^{\dagger} \Lambda^{\dagger} \delta & \delta^{\dagger} \delta
\end{array}\right]
$$

where $\Lambda \triangleq \Xi_{1}^{\dagger} \Xi_{2}$, and we use the fact that $\Xi_{1}^{\dagger} \Xi_{1}=\Xi_{2}^{\dagger} \Xi_{2}=\mathbf{I}$. For $k_{2}=0, l_{2} \neq 0, \boldsymbol{\Lambda}$ can be written as

$$
\boldsymbol{\Lambda}=\left(\mathbf{F}_{N}^{\dagger} \otimes \mathbf{I}_{M}\right)^{\dagger} \boldsymbol{\Pi}^{l_{2}}\left(\mathbf{F}_{N}^{\dagger} \otimes \mathbf{I}_{M}\right)
$$

Therefore, the determinant of $\Upsilon(\boldsymbol{\delta})$ is equal to

$$
\begin{aligned}
\operatorname{det}[\boldsymbol{\Upsilon}(\boldsymbol{\delta})] & =\left|\boldsymbol{\delta}^{\dagger} \boldsymbol{\delta}\right|^{2}-\left|\boldsymbol{\delta}^{\dagger} \boldsymbol{\Lambda} \boldsymbol{\delta}\right|^{2} \\
& =\left|\hat{\boldsymbol{\delta}}^{\dagger} \hat{\boldsymbol{\delta}}\right|^{2}-\left|\hat{\boldsymbol{\delta}}^{\dagger} \boldsymbol{\Pi}^{l_{2}} \hat{\boldsymbol{\delta}}\right|^{2}
\end{aligned}
$$

where $\hat{\boldsymbol{\delta}}=\left(\mathbf{F}_{N}^{\dagger} \otimes \mathbf{I}_{M}\right) \boldsymbol{\delta}$. From (19) and (7), using CauchySchwarz inequality, we can see that OTFS achieves rank 1 only when

$$
\hat{\boldsymbol{\delta}}=e^{j \theta} \boldsymbol{\Pi}^{l_{2}} \hat{\boldsymbol{\delta}}
$$

for some angle $\theta$. For simplicity, we assume $l_{2}$ divides $M$. Through simple algebraic computations, the condition in (20) can be written as

$$
\hat{\boldsymbol{\delta}}(r)=e^{j \theta} \hat{\boldsymbol{\delta}}\left(\left[r-l_{2}\right]_{M}\right), \text { for } 0 \leq r \leq M N-1,
$$

where $\theta$ is of the form $2 \pi \frac{l_{2}}{M N} c$, for any $c \in \mathbf{Z}$. As $\boldsymbol{\delta}=$ $\left(\mathbf{F}_{N}^{\dagger} \otimes \mathbf{I}_{M}\right)^{\dagger} \hat{\boldsymbol{\delta}}=\left(\mathbf{F}_{N} \otimes \mathbf{I}_{M}\right) \hat{\boldsymbol{\delta}}$, from (21), the value of $\boldsymbol{\delta}$ becomes

$$
\begin{aligned}
& {\left[\delta_{m, 0}, \delta_{m, 1}, \cdots, \delta_{m, N-1}\right]^{T}} \\
& =\mathbf{F}_{N}\left[e^{j p \theta} \hat{\boldsymbol{\delta}}(q), e^{j p \theta} e^{j \frac{M}{l_{2}} \theta} \hat{\boldsymbol{\delta}}(q), \cdots, e^{j p \theta} e^{j \frac{M}{l_{2}}(N-1) \theta} \hat{\boldsymbol{\delta}}(q)\right]^{T} \\
& =e^{j p \theta} \hat{\boldsymbol{\delta}}(q)[0, \cdots, \underbrace{1}_{\left(c^{\prime}+1\right)^{t h}}, 0 \text { entry }
\end{aligned}
$$

where $p=\left\lfloor m / l_{2}\right\rfloor, q=[m]_{l_{2}}, 0 \leq m \leq M-1$, and $c^{\prime}=[c]_{N}$. Therefore, the entries of $\delta$ can be written as

$$
\delta_{m, n}= \begin{cases}e^{j p \theta} \delta_{q, c^{\prime}+1}, & \text { if } n=c^{\prime}+1 \\ 0, & \text { otherwise. }\end{cases}
$$

for $0 \leq m \leq M-1$. However, as $\delta_{m, n} \in \mathbf{Z}[j]$, from (23) we can derive that $e^{j p \theta} \in \mathbf{Z}[j]$, which is possible only when $e^{j \theta}$ takes values from the set $\{+1,-1,+j,-j\}$.

Example 1: Consider $M=2, N=2, l_{2}=1, k_{2}=0$ and 4-QAM modulation. The following are some of the $\delta$ patterns that yield rank 1:

$$
\begin{aligned}
& {[2,2,0,0]^{T},[2,-2,0,0]^{T},[-2,2,0,0]^{T},[-2,-2,0,0]^{T},} \\
& {[0,0,2,2 j]^{T},[0,0,2 j,-2]^{T},[0,0,-2,2 j]^{T},[0,0,-2,-2 j]^{T}}
\end{aligned}
$$

We can clearly see that all the above patterns follow the structure in (23).

1) Upper bound on the number of pairs yielding rank 1: From (23), for a given $\mathbf{x}$, the maximum number of $\hat{\mathbf{x}}$ that yield rank 1 can be computed as $4 N\left(Q^{l_{2}}-1\right)$, where $N$ denotes the all possible values of $c^{\prime},\left(Q^{l_{2}}-1\right)$ is the number of possible selection of QAM symbols for a given $c^{\prime}$ which is due to $0 \leq q \leq l_{2}-1$, and 4 is due to the set $\{+1,-1,+j,-j\}$.

For the case of $l_{2}$ not dividing $M$, the value of $l_{2}$ in the condition (21) is replaced by $l_{2}^{\prime}=\operatorname{gcd}\left(l_{2}, M\right)$.

C. $l_{2}=0$ and $k_{2} \neq 0$

For the case of $l_{2}=0$ and $k_{2} \neq 0$, the value of $\Lambda$ in (16) becomes

$$
\boldsymbol{\Lambda}=\left(\mathbf{F}_{N}^{\dagger} \otimes \mathbf{I}_{M}\right)^{\dagger} \boldsymbol{\Delta}^{k_{2}}\left(\mathbf{F}_{N}^{\dagger} \otimes \mathbf{I}_{M}\right)
$$

Now, the determinant of $\Upsilon(\boldsymbol{\delta})$ can be written as

$$
\operatorname{det}[\boldsymbol{\Upsilon}(\boldsymbol{\delta})]=\left|\hat{\boldsymbol{\delta}}^{\dagger} \hat{\boldsymbol{\delta}}\right|^{2}-\left|\hat{\boldsymbol{\delta}}^{\dagger} \boldsymbol{\Delta}^{k_{2}} \hat{\boldsymbol{\delta}}\right|^{2}
$$

where $\hat{\boldsymbol{\delta}}=\left(\mathbf{F}_{N}^{\dagger} \otimes \mathbf{I}_{M}\right) \boldsymbol{\delta}$. Therefore, using Cauchy-Schwarz inequality, we can see that OTFS achieves rank 1 only when

$$
\hat{\boldsymbol{\delta}}=e^{j \theta} \boldsymbol{\Delta}^{k_{2}} \hat{\boldsymbol{\delta}}
$$

for some angle $\theta$. For simplicity, we assume $k_{2}$ divides $N$. Assuming $\hat{\boldsymbol{\delta}}(r) \neq 0$ for some $0 \leq r \leq M N-1$, from (26), we can obtain the conditions on $\hat{\boldsymbol{\delta}}$ as

$$
\hat{\boldsymbol{\delta}}\left(r^{\prime}\right)=0 \text { for }\left[r^{\prime}-r\right]_{\frac{M N}{k_{2}}} \neq 0
$$


That is, $\hat{\boldsymbol{\delta}}$ contains at the most $k_{2}$ non-zero elements separated by $\frac{M N}{k_{2}}$. Now, similar to (22), the value of $\delta$ can be written as

$$
\begin{aligned}
& {\left[\delta_{m, 0}, \delta_{m, 1}, \cdots, \delta_{m, N-1}\right]^{T}} \\
& =\mathbf{F}_{N}[\hat{\boldsymbol{\delta}}(m), \hat{\boldsymbol{\delta}}(m+M), \cdots, \hat{\boldsymbol{\delta}}(m+(N-1) M)]^{T}
\end{aligned}
$$

From (27), we can immediately see that the vector in (28) can have at the most $k_{2}$ non-zero elements for one value of $m$, $0 \leq m \leq M-1$, and all zero elements for all other $m$.

Example 2: Consider $M=2, N=2, l_{2}=0, k_{2}=1$ and 4-QAM modulation. As $k_{2}=1$, only one element in (28) can be non-zero for some value of $m=m^{\prime}$. Therefore, the entries of $\delta$ can be written as

$$
\delta_{m, n}= \begin{cases}e^{-j 2 \pi \frac{n c}{N}} \delta_{m^{\prime}, n}, & \text { if } m=m^{\prime} \\ 0, & \text { otherwise }\end{cases}
$$

for $0 \leq n, c \leq N-1$. The following are some of the $\delta$ patterns that yield rank 1 :

$$
\begin{aligned}
& {[2,0,2,0]^{T},[-2,0,-2,0]^{T},[-2,0,2,0]^{T},[2,0,-2,0]^{T},} \\
& {[0,-2 j, 0,2 j]^{T},[0,2 j, 0,2 j]^{T},[0,2 j, 0,-2 j]^{T},[0,2,0,-2]^{T},}
\end{aligned}
$$

We can clearly see that all the above patterns follow the structure in (29).

1) Upper bound on the number of pairs yielding rank 1: From (28), for a given $\mathbf{x}$, the maximum number of $\hat{\mathbf{x}}$ yielding rank 1 can be computed as $M \frac{N}{k_{2}}\left(Q^{k_{2}}-1\right)$, where $M$ denotes the all possible values of $m,\left(Q^{k_{2}}-1\right)$ is the number of possible selection of QAM symbols for a given $m$ which is due to the $k_{2}$ non-zero elements in (28), and $\frac{N}{k_{2}}$ is the possible $k_{2}$ non-zero sets in (28). For the case of $k_{2}$ not dividing $N$, the value of $k_{2}$ in (27) is replaced by $k_{2}^{\prime}=\operatorname{gcd}\left(k_{2}, N\right)$ and $\hat{\boldsymbol{\delta}}$ can contain at the most $k_{2}^{\prime}$ non-zero elements.

\section{Diversity for the three cases above}

Summarizing our analysis of the three cases above, an upper bound $\mathcal{N}(\mathbf{x}, \hat{\mathbf{x}})$ to the number of pairs $(\mathbf{x}, \hat{\mathbf{x}})$ yielding rank 1 is

$$
\mathcal{N}(\mathbf{x}, \hat{\mathbf{x}})= \begin{cases}0, & \text { if } l_{2} \neq 0, k_{2} \neq 0 \\ 4 N\left(Q^{l_{2}^{\prime}}-1\right) Q^{M N}, & \text { if } l_{2} \neq 0, k_{2}=0 \\ M \frac{N}{k_{2}^{\prime}}\left(Q^{k_{2}^{\prime}}-1\right) Q^{M N}, & \text { if } l_{2}=0, k_{2} \neq 0\end{cases}
$$

Now, consider the ratio of $\mathcal{N}(\mathbf{x}, \hat{\mathbf{x}})$ to the total number of possible pairs $\mathcal{T}(\mathbf{x}, \hat{\mathbf{x}})=Q^{M N}\left(Q^{M N}-1\right)$. we obtain

$$
\mathcal{R}(\mathbf{x}, \hat{\mathbf{x}})=\frac{\mathcal{N}(\mathbf{x}, \hat{\mathbf{x}})}{\mathcal{T}(\mathbf{x}, \hat{\mathbf{x}})} \approx \begin{cases}0, & \text { if } l_{2} \neq 0, k_{2} \neq 0 \\ \frac{4 N}{Q^{\left(M N-l_{2}^{\prime}\right)}}, & \text { if } l_{2} \neq 0, k_{2}=0 \\ \frac{M N}{k_{2}^{\prime} Q^{\left(M N-k_{2}^{\prime}\right)}}, & \text { if } l_{2}=0, k_{2} \neq 0\end{cases}
$$

As $l_{2} \ll M, k_{2} \ll N$, we can see that, as $M$ and $N$ grow larger, $\mathcal{R}(\mathbf{x}, \hat{\mathbf{x}})$ quickly approaches zero.

$$
\mathcal{R}(\mathbf{x}, \hat{\mathbf{x}}) \stackrel{M, N \rightarrow \infty}{\longrightarrow} 0
$$

Example 3: Consider $M=16, N=16, l_{2}=4, k_{2}=0$, and 4-QAM modulation. Then the value of $\mathcal{R}(\mathbf{x}, \hat{\mathbf{x}})$ is $\approx 10^{-150}$.

Now, consider again the union bound to $P_{\mathrm{e}}$, as shown in (6). Each individual term in sum (6) can be viewed as a function of SNR whose slope is essentially dictated by the value of $\rho(\mathbf{x}$, ' $\mathbf{x})$. As SNR increases, the terms with higher values of $\rho(\mathbf{x}$, $\mathbf{x}^{\wedge}$ ) become increasingly less relevant to determine the value of $P_{\mathrm{e}}$, so that for very high SNR the only significant terms in (6) are those with the lowest value of $\rho\left(\mathbf{x}, \mathbf{x}^{\wedge}\right)$. This justifies the use of the standard definition (7) of diversity $\rho$, useful for very high SNR. For intermediate values of SNR, however, the use of standard diversity might not be appropriate whenever the number of terms in (6) with exponent higher than $\rho$ is much greater than the number of those with expo-nent $\rho$. If this is the case, for those values of SNR the slope of the union-bound curve might be dictated by an exponent higher than $\rho$. We call this exponent "effective diversity." In our context, OTFS achieves full effective diversity with typical system parameters as $P=2$. Future work will show the extension of the above analysis to $P$ values greater than 2 .

Remark 1: Note that the above diversity analysis differs from the work in [8] in two aspects.

1) The diversity analysis in [8] is proposed only for the ideal biorthogonal waveforms that satisfy both time and frequency orthogonality conditions, which is not practically feasible due to Heisenberg's uncertainty principle. Instead, the analysis in our work assumes feasible rectangular waveforms, which yield different input-output relations (extra phase shifts in (7)) compared to the ideal waveforms [5], [8]. Moreover, our analysis developed for rectangular waveforms can be straightforwardly extended to any arbitrary waveforms, for example, raised cosine waveforms [5].

2) The work in [8] showed that the standard diversity order of the OTFS system is one and proposed a phase rotation based precoding scheme to achieve full diversity. However, in this work, we show that, with sufficiently large $M$ and $N$, the OTFS system achieves full effective diversity, thus any precoding schemes are not required.

\section{Simulation Results}

In this section, we present the BER of OTFS for different choices of system parameters. We consider $P=2, l_{1}=$ $0, k_{1}=0$, and 4-QAM modulation in all the simulations. The path coefficients are $h_{1}, h_{2} \sim \mathcal{C N}(0,1 / 2)$. Figs. 2a, $2 \mathrm{~b}$, and 3 show the BER of OTFS for different $\left(l_{2}, k_{2}\right)$ with $(M=2, N=2),(M=4, N=4)$, and $(M=16, N=16)$ respectively. We use the optimal ML detector in Figs. 2a and $2 \mathrm{~b}$ and the message passing detector [7] in Fig. 3. Note that the plots of $c_{1}(\mathrm{SNR})^{-2}$ and $c_{2}(\mathrm{SNR})^{-1}$ are only used to identify the slope of the curves, and do not represent an upper bound. From the figures, we can observe that:

i) In all the three cases, OTFS achieves the full diversity of two with the second path located at $l_{2}=1, k_{2}=1$. This is due to the absence of rank 1 pairs if $k_{2} \neq k_{1}$ and $l_{2} \neq l_{1}$. 


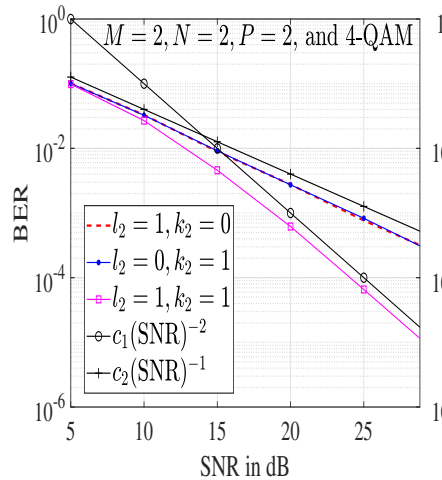

(a) $M=2, N=2$

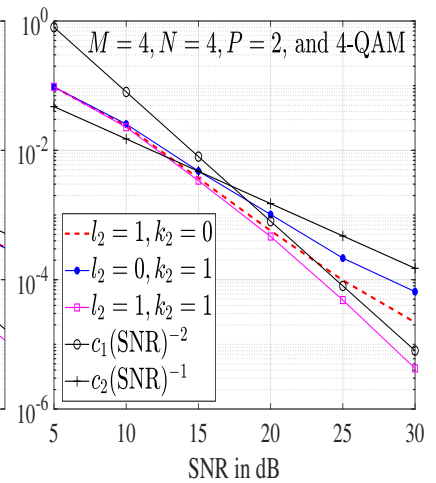

(b) $M=4, N=4$
Fig. 2. BER of OTFS for different $\left(l_{2}, k_{2}\right)$ with $P=2$ and 4-QAM.

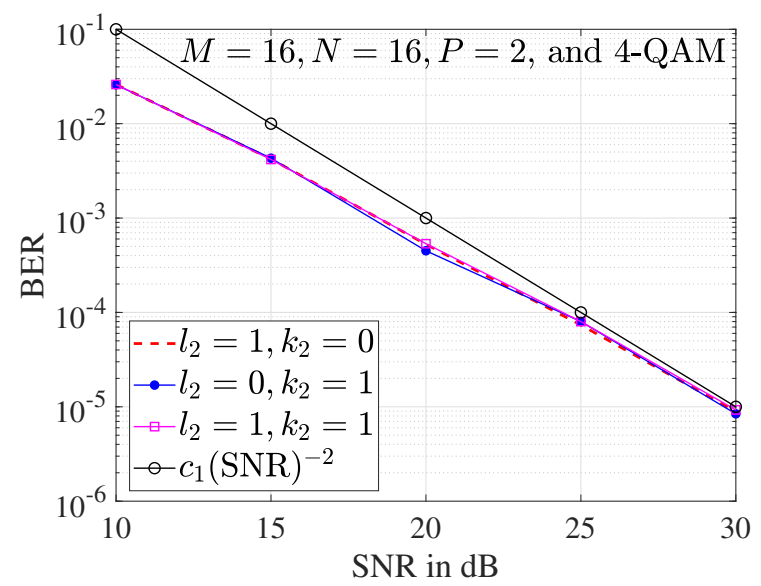

Fig. 3. BER of OTFS for different $\left(l_{2}, k_{2}\right)$ with $M=16, N=16, P=2$, and 4-QAM.

ii) OTFS achieves rank 1 with the second paths located at $l_{2}=1, k_{2}=0$ or $l_{2}=0, k_{2}=1$ for $(M=2, N=2)$ and $(M=4, N=4)$. This is because of the upper bound in (30) is significant for these system parameters. However, we can see that the diversity change happens at a higher SNR with ( $M=4, N=4)$ compared to the case of $(M=2, N=2)$.

iii) Finally, independent of the second path location, OTFS achieves the same performance for $(M=16, N=16)$ for the BER ranges up to $10^{-5}$. This is justified by the small number of rank-1 pairs as mentioned in Example 3.

From the above observations, we conclude that OTFS system achieves full effective diversity for sufficiently large values of $M$ and $N$.

\section{CONClusion}

We have analyzed the diversity of OTFS over two-path channels. After deriving an upper bound on the number of pairs that prevent the achievement of full rank, we have shown that the number of these pairs is relatively vanishingly small for sufficiently large values of $M$ and $N$ (e.g., $M=N=16$ ). Through analysis and simulations, we can conclude that, even though the theoretical diversity of OTFS is one, effective diversity, which takes value 2 , is the significant parameter expressing error performance.

\section{REFERENCES}

[1] E. Biglieri, Coding for Wireless Channels. New York: Springer, 2005.

[2] R. Hadani, S. Rakib, M. Tsatsanis, A. Monk, A. J. Goldsmith, A. F. Molisch, and R. Calderbank, "Orthogonal time frequency space modulation," in Proc. IEEE WCNC, San Francisco, CA, USA, March 2017.

[3] R. Hadani, S. Rakib, S. Kons, M. Tsatsanis, A. Monk, C. Ibars, J. Delfeld, Y. Hebron, A. J. Goldsmith, A.F. Molisch, and R. Calderbank, "Orthogonal time frequency space modulation," Available online: https://arxiv.org/pdf/1808.00519.pdf.

[4] R. Hadani and A. Monk, "OTFS: A new generation of modulation addressing the challenges of 5G," OTFS Physics White Paper, Cohere Technologies, 7 Feb. 2018. Available online: https://arxiv.org/pdf/1802.02623.pdf.

[5] P. Raviteja, Y. Hong, E. Viterbo, and E. Biglieri, "Practical pulse-shaping waveforms for reduced-cyclic-prefix OTFS," IEEE Trans. Veh. Technol., vol. 68, no. 1, pp. 957-961, Jan. 2019.

[6] E. Biglieri, P. Raviteja, and Y. Hong, "Error performance of orthogonal time frequency space (OTFS) modulation," in Proc. IEEE ICC Workshop 2019, Shanghai, China, May 2019.

[7] P. Raviteja, K. T. Phan, Y. Hong, and E. Viterbo, "Interference cancellation and iterative detection for orthogonal time frequency space modulation," IEEE Trans. Wireless Commun., vol. 17, no. 10, pp. 65016515, Oct. 2018.

[8] G. D. Surabhi, R. M. Augustine, and A. Chockalingam, "On the diversity of uncoded OTFS modulation in doubly-dispersive channels," IEEE Trans. Wireless Commun., vol. 18, no. 6, pp. 3049-3063, June 2019.

[9] K. R. Murali and A. Chockalingam, "On OTFS modulation for highDoppler fading channels," in Proc. ITA'2018, San Diego, Feb. 2018.

[10] L. Li, H. Wei, Y. Huang, Y. Yao, W. Ling, G. Chen, P. Li, and Y. Cai, "A simple two-stage equalizer With simplified orthogonal time frequency space modulation over rapidly time-varying channels," available online: https://arxiv.org/abs/1709.02505.

[11] T. Zemen, M. Hofer, D. Loeschenbrand, and C. Pacher, "Iterative detection for orthogonal precoding in doubly selective channels," in Proc. 2018 IEEE 29th Annual International Symposium on PIMRC, Bologna, pp. 1-7, Sept. 2018.

[12] A. Farhang, A. Rezazadeh, Reyhani, L. E. Doyle, and B. FarhangBoroujeny, "Low complexity modem structure for OFDM-based orthogonal time frequency space modulation," IEEE Wireless Commun. Lett., vol. 7, no. 3, pp. 344-347, June 2018.

[13] A. RezazadehReyhani, A. Farhang, M. Ji, R. R. Chen, and B. FarhangBoroujeny, "Analysis of discrete-time MIMO OFDM-based orthogonal time frequency space modulation," in Proc. 2018 IEEE ICC, Kansas City, MO, USA, pp. 1-6, May 2018.

[14] V. Khammammetti and S. K. Mohammed, "OTFS-based multiple-access in high Doppler and delay spread wireless channels," IEEE Wireless Commun. Lett., vol. 8, no. 2, pp. 528-531, April 2019. 